\title{
Effects of reduced food intake for 4 weeks on physiological parameters in toxicity studies in dogs
}

\author{
Junya Morita, Tomoko Izumi, Bunichiro Ogawa, Yoshiki Ban, Hironori Takagi, \\ Minoru Sasaki and Shunsuke Tsutsumi \\ Drug Safety, Drug Safety and Pharmacokinetics Laboratories, Taisho Pharmaceutical Co., Ltd., \\ 1-403 Yoshino-cho, Kita-ku, Saitama-shi, Saitama 331-9530, Japan
}

(Received September 23, 2016; Accepted October 28, 2016)

\begin{abstract}
This study sought to clarify the effects of reduced feeding on physiological parameters in dogs to enable appropriate evaluations of the safety and toxicity of test compounds. We measured alkaline phosphatase isozymes and the circulating blood volume, as well as clinical signs, body weight, hematology, blood chemistry, electrocardiography, organ weight, and histopathology, in male beagle dogs fed a diet consisting of $300 \mathrm{~g} /$ day or $150 \mathrm{~g} /$ day for 4 weeks. There were no abnormal clinical signs in any of the dogs. In the $150-\mathrm{g} /$ day feeding group, a decreased alkaline phosphatase 3 suggesting effects on the bone and a decreased circulating blood volume associated with body weight loss were observed. Additionally, the following changes were also observed in the 150-g/day group: a decrease in body weight; hematologic changes including decreases in white blood cells, neutrophils, red blood cells, hemoglobin, hematocrit and reticulocytes; blood chemical changes including decreases in aspartate aminotransferase, lactate dehydrogenase and calcium and an increase in the creatinine at week 1 or thereafter; electrocardiographic changes including a decrease in the heart rate, a prolonged QRS duration and the occurrence of a seconddegree atrioventricular block at week 3 or thereafter; and pathological changes including decreases in the weights of the liver and thymus, a decrease in hepatocyte rarefaction, and thymic atrophy. These results provide useful information for assessing the safety of compounds in toxicological studies, enabling direct treatment effects and secondary changes caused by decreased food intake to be distinguished.
\end{abstract}

Key words: Food reduction, Physiological parameters, Dogs, Toxicity study

\section{INTRODUCTION}

In toxicity studies, it is important to distinguish the treatment-related effects of test compounds from the influences of other factors. A treatment-related decrease in food intake is often observed in repeated-dose animal toxicity studies, and this decrease can exert secondary effects on the toxicological parameters. In dogs, we previously reported that a reduced food intake for 12 weeks affected many physiological parameters commonly measured in toxicity studies (Morita et al., 2015), resulting in decreases in body weight, alkaline phosphatase (ALP) level, leukocyte count, and heart rate (HR). However, the effects of a decreased food intake on some parameters, such as the effect of a decreased ALP level on bone growth and the effects of a diet-induced decrease in body weight on the circulating blood volume, remain unclear. Therefore, we measured the levels of ALP isozymes and the circulat- ing blood volume and performed pathological examinations of several organs, including bone, in dogs subjected to reduced feeding. Toxicity parameters, including body weight and hematologic, blood chemistry and electrocardiographic parameters, were also determined once a week to confirm whether these parameters were influenced by shorter periods of reduced feeding.

The purpose of the present study was to clarify the effects of reduced food intake on physiological parameters in dogs over a 4-week period to enable appropriate assessments of the safety and toxicity of test compounds in general toxicity studies performed using dogs.

\section{MATERIALS AND METHODS}

\section{Animals and housing conditions}

Ten male beagle dogs from Covance Research Products Inc. (Cumberland, VA, USA) were used. The animals

Correspondence: Junya Morita (E-mail: ju-morita@so.taisho.co.jp) 


\section{J. Morita et al.}

were housed individually in cages in an air-conditioned animal room, where the room temperature $\left(23 \pm 5^{\circ} \mathrm{C}\right)$, relative humidity $(50 \pm 20 \%)$ and light and dark cycle (12 hr each, lights on from 07:15 to 19:15) were controlled. During the 5-week pre-test period, all the dogs were fed a canine diet (DS-A; $360 \mathrm{kcal} / 100 \mathrm{~g}$; Oriental Yeast Co., Ltd., Tokyo, Japan). The dogs were randomly divided into 2 groups, a control group and a low feeding (LF) group, balanced according to baseline body weight, with 5 animals placed in each group. None of the animals were littermates. At the beginning of the reduced feeding period, the animals were 9 months old. All the dogs were fed daily from 09:00 to 14:00. Tap water was made available ad libitum.

All the experimental procedures involving the animals were performed with the approval of the Institutional Animal Care and Use Committee of Taisho Pharmaceutical Co., Ltd., and were in accordance with the Guidelines for Proper Conduct of Animal Experiments (Science Council of Japan, 2006).

\section{Study design}

The experimental design is shown in Fig. 1. The experimental period simulated a 4-week toxicity study in dogs, consisting of a 5-week pre-test period before the group assignment and a 4 -week reduced feeding period. A reduced feeding period of 4 weeks was selected because changes in parameters related to a reduced food intake, such as a decreased body weight, ALP level, leukocyte count and HR, were observed at week 4 in a previous 12-week study (Morita et al., 2015).

All the dogs were fed $300 \mathrm{~g} /$ day of a canine diet during the pre-test period. This amount was considered to be sufficient based on the reported energy requirements of beagle dogs (Durrer and Hannon, 1962) and is the amount usually provided in general toxicity studies performed in dogs so as not to affect evaluations of the test compounds. During the reduced feeding period, dogs in the LF group received $150 \mathrm{~g} /$ day, based on the results of a previous study (Morita et al., 2015). Dogs in the control group were fed $300 \mathrm{~g} /$ day throughout the experimental period.

\section{Examinations and methods}

The clinical signs and food consumption were monitored daily for all the animals. The body weight and hematology, blood chemistry, ALP isozymes and electrocardiography parameters were measured during the pretest period and at weeks 1, 2, 3 and 4 (W1, W2, W3 and W4) during the reduced feeding period. The circulating blood parameters were measured during the pre-test period and at W4. All the animals were necropsied at the end of W4.

Blood samples were collected from the cephalic vein after an overnight fast. The samples were placed in EDTA-2K-treated tubes for the hematologic evaluation, in $3.2 \%$ trisodium citrate dihydrate solution-added tubes for the evaluation of coagulation parameters, in plasma separator tubes for the measurement of lactate dehydrogenase (LDH), and in serum separator tubes for the evaluation of ALP isozymes and blood chemistry parameters other than LDH. All the sample tubes other than the EDTA-2Ktreated tubes were centrifuged (approximately $1200 \times \mathrm{g}$, $4^{\circ} \mathrm{C}, 15 \mathrm{~min}$ ) to obtain plasma or serum. Hematological

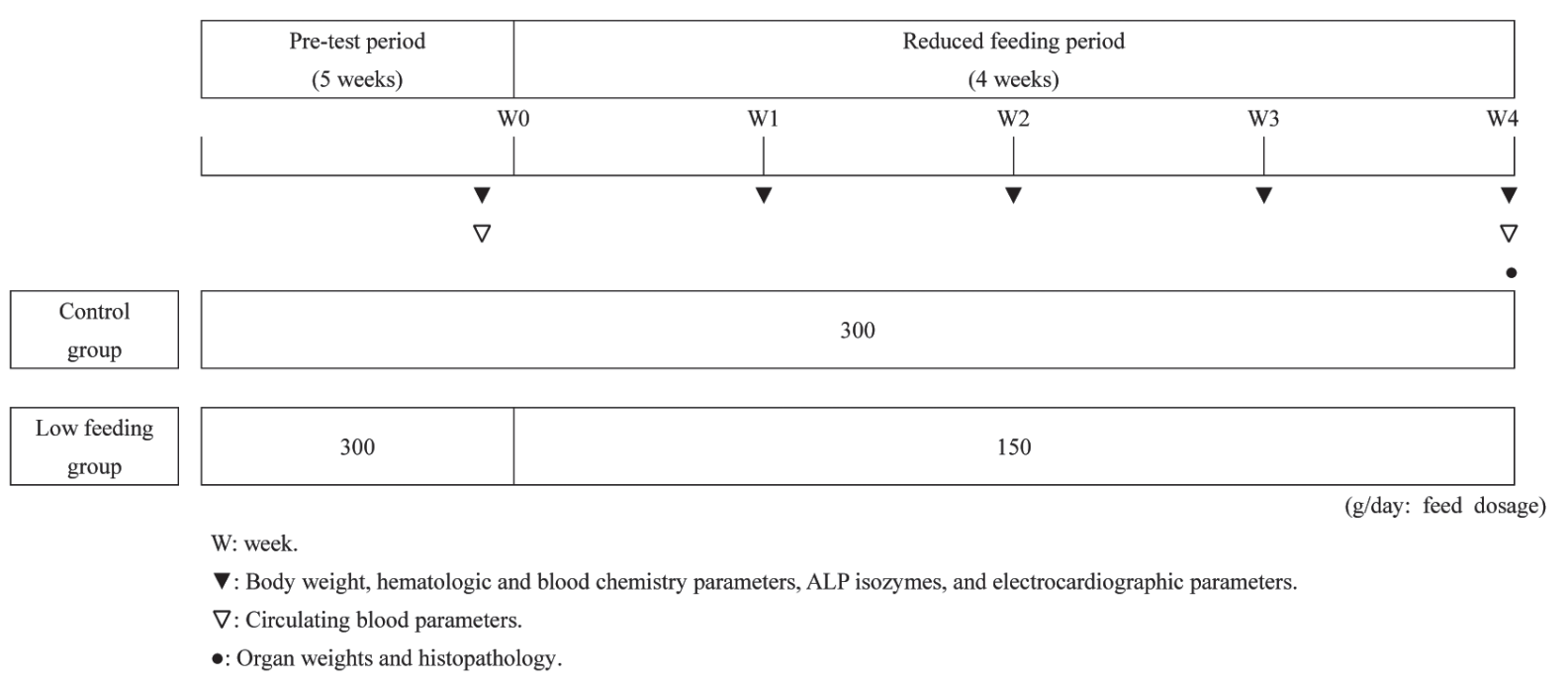

Fig. 1. Experimental design. 
Effects of reduced food intake in dogs

parameters including the white blood cell (WBC) count, differential WBC absolute counts (neutrophils [Neut], lymphocytes [Lymp], monocytes [Mono], eosinophils [Eos] and basophils [Baso]), red blood cell (RBC) count, hemoglobin (HGB) level, hematocrit (HCT) level, reticulocyte (Retic) count, mean corpuscular volume (MCV), mean corpuscular hemoglobin $(\mathrm{MCH})$ level, mean corpuscular hemoglobin concentration $(\mathrm{MCHC})$ and platelet (PLT) count were assayed using a hematology system (ADVIA 120; Siemens Healthcare Diagnostics K.K., Tokyo, Japan). Coagulation parameters including the prothrombin time (PT) and activated partial thromboplastin time (APTT) were assayed using an automated blood coagulation analyzer (CS-2000i; Sysmex Corporation, Hyogo, Japan). Blood chemistry parameters including the levels of aspartate aminotransferase (AST), alanine aminotransferase (ALT), ALP, LDH, $\gamma$-glutamyl transpeptidase $(\gamma$-GTP), total bilirubin (TBIL), glucose (GLU), total protein (TP), albumin (ALB), albumin/globulin ratio $(\mathrm{A} / \mathrm{G})$, total cholesterol (TCHO), triglyceride ( $\mathrm{TG})$, free fatty acid (FFA), phospholipid (PL), urea nitrogen (UN), creatinine (CRE), calcium $(\mathrm{Ca})$, inorganic phosphorus (IP), sodium $(\mathrm{Na})$, potassium $(\mathrm{K})$ and chloride $(\mathrm{Cl})$ were assayed using a clinical analyzer (7180; Hitachi HighTechnologies Corporation, Tokyo, Japan). ALP isozymes (ALP1, 2, 3 and 5) were assayed using an electrophoresis analyzer (REP; Helena Laboratories Japan Co., Ltd., Saitama, Japan).

Electrocardiography was performed without the use of anesthesia. The electrocardiographic parameters including waveforms, HR, PR interval, QRS duration and QT interval were measured (D700, FUKUDA M-E KOGYO Co., Ltd., Tokyo, Japan) and the QTc was calculated using Matsunaga's formula (Matsunaga et al., 1997).

The circulating blood parameters were measured according to the following method using indocyanine green (ICG, diagnogreen for injection; Daiichi Sankyo Co., Ltd., Tokyo, Japan): $5 \mathrm{mg}$ of ICG was administered as an intravenous bolus into the saphenous vein. Blood samples were collected from the cephalic vein at 5,15 and 25 min after administration and placed in plasma separator tubes. The sample tubes were centrifuged (approximately $1200 \times \mathrm{g}, 4^{\circ} \mathrm{C}, 15 \mathrm{~min}$ ) to obtain plasma. The light absorption of the plasma was measured using a spectrophotometer (xMark; Bio-Rad Laboratories, Inc., Hercules, CA, USA) at an absorption wavelength of $805 \mathrm{~nm}$ and a reference wavelength of $900 \mathrm{~nm}$. The plasma concentrations of ICG were calculated from a calibration curve. The initial plasma concentration $\left(\mathrm{C}_{0}\right)$ of ICG was obtained by extrapolation using a single logarithmic chart. The following formulas were used to calculate the circulating plasma volume and the circulating blood volume, and the circulating blood volume per body weight ratio was calculated.

$$
\begin{aligned}
\text { Circulating plasma volume }= & \mathrm{ICG} \text { dose } / \mathrm{C}_{0} \\
\text { Circulating blood volume }= & \text { Circulating plasma volume } / \\
& (1-\mathrm{HCT})
\end{aligned}
$$

At the end of W4, the dogs were anesthetized with the injection of pentobarbital sodium (somnopentyl; Kyoritsu Seiyaku Corporation, Tokyo, Japan) into the cephalic vein and were subsequently euthanized by exsanguination, then necropsied. The weights of the following organs were measured: brain, pituitary, heart, lung, liver, kidney, spleen, thymus, thyroid, adrenal, submandibular gland, testis, epididymis and prostate. The following organs were collected at necropsy: brain, pituitary, heart, lung, liver, gallbladder, kidney, spleen, thymus, adrenal, thyroid, parathyroid, submandibular gland, sublingual gland, parotid gland, testis, epididymis, prostate, tongue, esophagus, stomach, duodenum, jejunum, ileum, colon, rectum, mesenteric lymph node, pancreas, skeletal muscle, femur, sternum, bone marrow, spinal cord, sciatic nerve, skin, mammary gland, eyeball, optic nerve, urinary bladder, trachea and aorta. The testes and epididymides were first fixed in Bouin's solution and then fixed in $10 \%$ neutral buffered formalin. The eyeballs were prefixed in formaldehyde-glutaraldehyde solution and then immersed in 10\% neutral buffered formalin. The femur and sternum were fixed in $10 \%$ neutral buffered formalin and were decalcified using the rapid decalcification method (Plank-Rychlo's method). The remaining organs that were collected at necropsy were fixed in $10 \%$ neutral buffered formalin. After fixation, the tissues were embedded in paraffin, stained with hematoxylin and eosin, and examined microscopically.

\section{Statistical analysis}

The mean values and standard deviations in the body weights and hematology, blood chemistry, ALP isozymes, electrocardiography, circulating blood and organ weight parameters were calculated for each study week in both the control and LF groups.

Pairwise comparisons between the control and LF groups were made to analyze the changes under the same experimental conditions. First, the homogeneity of variance was analyzed using an F-test for each interval. If the variance was homogeneous, a Student $t$-test was performed. On the other hand, if the variance was heterogeneous, the Aspin-Welch's t-test was performed. Also, to analyze individual differences, comparisons of time-series data were made: a paired t-test with Bonferroni correction 


\section{J. Morita et al.}

was performed to determine the significance of differences in each group between the pre-test values and the values at time-points during the reduced feeding period.

The F-test was applied with a significance level set at $5 \%$ (two-tailed), while the significance levels for the other tests were set at $1 \%$ and $5 \%$ (two-tailed). Changes in clinical signs, food consumption or histopathology were not analyzed statistically.

\section{RESULTS}

\section{Clinical signs, food consumption and body weights}

No abnormal changes in clinical signs were observed at any time in any of the dogs during the experimental period.

All the animals consumed almost $100 \%$ of their daily feed dosage in both the control and LF groups during the experimental period.

The mean body weight changes are shown in Fig. 2. In the LF group, significant decreases $(P<0.05$ and/or 0.01$)$ in body weight were observed throughout the reduced feeding period, compared with the values in the control group and/or the pre-test values. In the LF group, the average percent changes in body weight at W1, W2, W3 and $\mathrm{W} 4$ were $-5.0 \%,-8.4 \%,-12.3 \%$ and $-14.5 \%$ relative to the pre-test value, respectively.

\section{Hematology}

Changes in the hematologic parameters are shown in

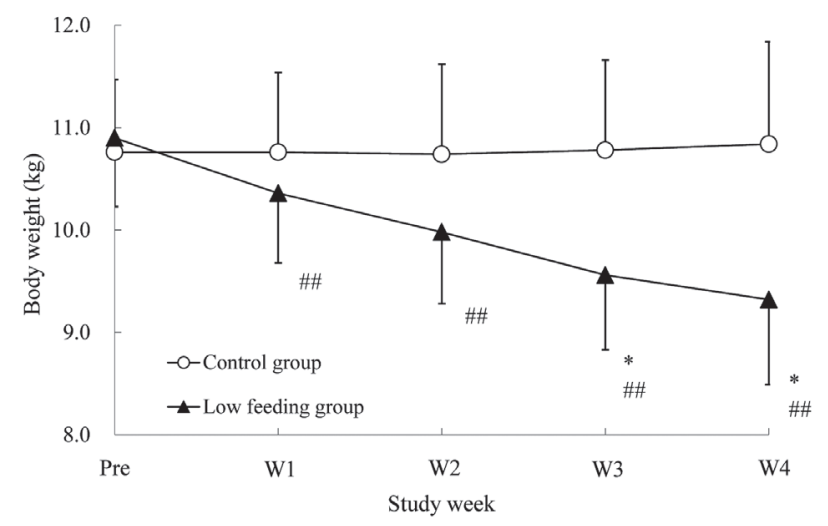

Fig. 2. Body weight changes. Data from the control group and the low feeding (LF) group are shown as the mean + S.D. and the mean - S.D., respectively $(\mathrm{n}=5)$. ${ }^{*} P<$ 0.05 for comparison with the control group using the Student $t$-test. $\# \# P<0.01$ for comparison with the pretest value in each group using a paired $t$-test with Bonferroni correction.
Table 1. The WBC and Neut counts in the LF group were significantly lower $(P<0.05)$ than those in the control group at W2. In the LF group, the average percent changes in the WBC and Neut counts at W2 were $-19.5 \%$ and $-27.5 \%$ relative to the pre-test values, respectively.

The RBC count and the HGB and HCT levels in the LF group were significantly lower $(P<0.01)$ than the pre-test values at $\mathrm{W} 1, \mathrm{~W} 2, \mathrm{~W} 3$ and/or W4. In the LF group, the average percent changes in the RBC count and the HGB and HCT levels at W4 were $-14.9 \%,-16.5 \%$ and $-14.3 \%$ relative to the pre-test values, respectively. The Retic counts in the LF group were significantly lower $(P<0.05$ and/or 0.01) than that in the control group and/ or the pre-test values throughout the reduced feeding period. In the LF group, the average percent change in the Retic count at W4 was $-67.7 \%$ relative to the pre-test value.

\section{Blood chemistry}

Changes in the blood chemistry parameters are shown in Table 2. In the LF group, the AST level was significantly lower $(P<0.05$ and/or 0.01$)$ than that in the control group and/or the pre-test value, and the ALP level was significantly lower $(P<0.05$ or 0.01$)$ than the pretest value throughout the reduced feeding period. The $\mathrm{Ca}$ level was significantly lower $(P<0.05$ and/or 0.01$)$ in the LF group than that in the control group and/or the pretest value at W2, W3 and W4. In the LF group, the average percent changes in the AST, ALP and Ca levels at W4 were $-25.4 \%,-36.8 \%$ and $-7.2 \%$ relative to the pre-test values, respectively. Furthermore, the LDH level at W2 in the LF group was significantly lower $(P<0.05$ or 0.01$)$ than that in the control group and the pre-test value, while the CRE level at W1 and W2 was significantly higher $(P<0.05)$ than that in the control group and/or the pretest value. In the LF group, the average percent changes in the LDH and CRE levels at W2 were $-40.0 \%$ and $+11.0 \%$ relative to the pre-test values, respectively.

\section{ALP isozymes}

Changes in the ALP isozyme levels are shown in Table 3. In the LF group, decreases in the ALP3 level were observed throughout the reduced feeding period, and significant differences $(P<0.05$ or 0.01$)$ compared to the pre-test value were observed at W1, W2 and W4. In the LF group, the average percent changes in the ALP3 level at $\mathrm{W} 1, \mathrm{~W} 2, \mathrm{~W} 3$ and $\mathrm{W} 4$ were $-25.2 \%,-35.9 \%,-29.7 \%$ and $-35.8 \%$ relative to the pre-test value, respectively. The ALP1, 2 and 5 levels in the LF group were comparable to the pre-test values and the values in the control group throughout the reduced feeding period. 
Effects of reduced food intake in dogs

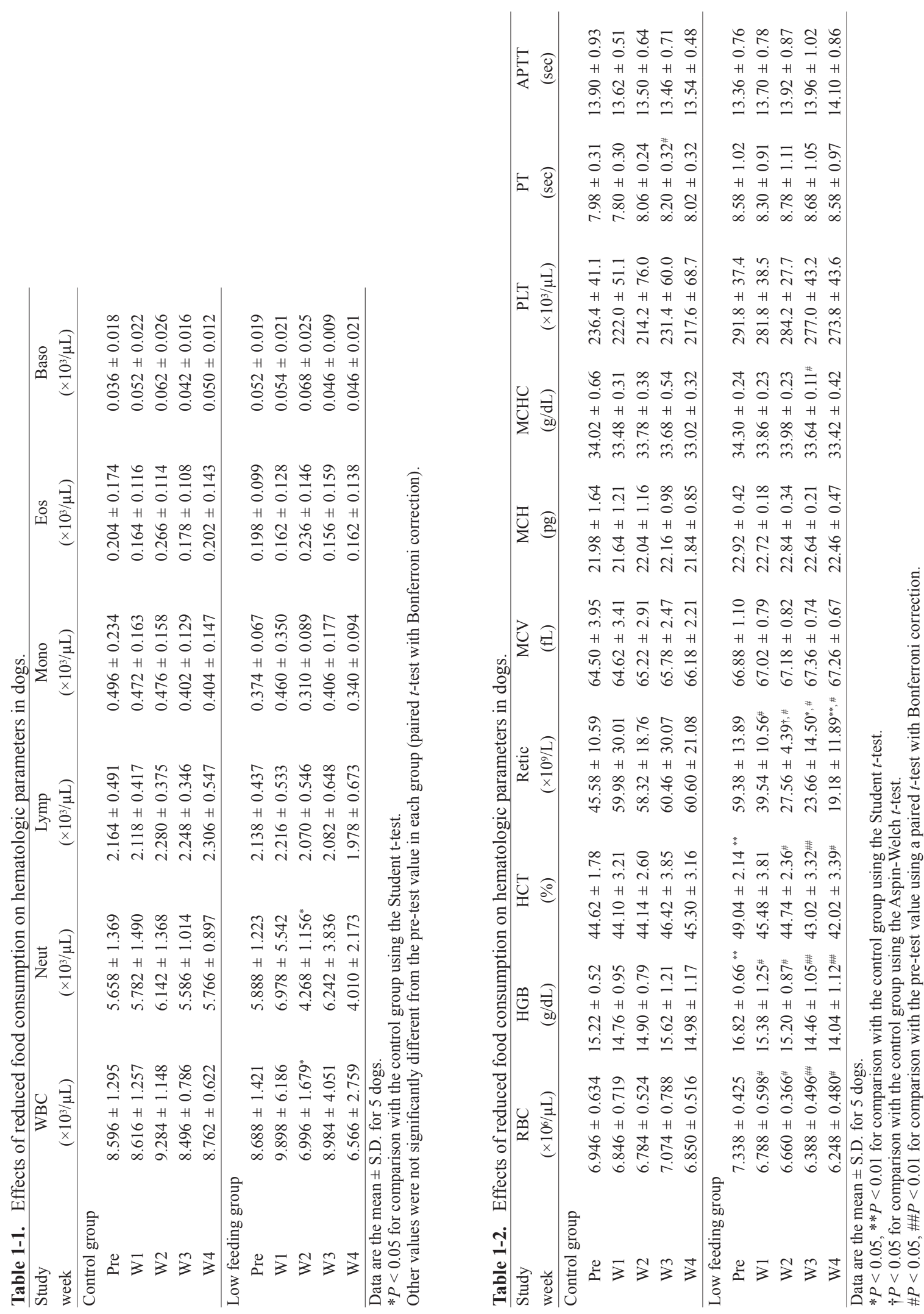

Vol. 42 No. 1 
J. Morita et al.
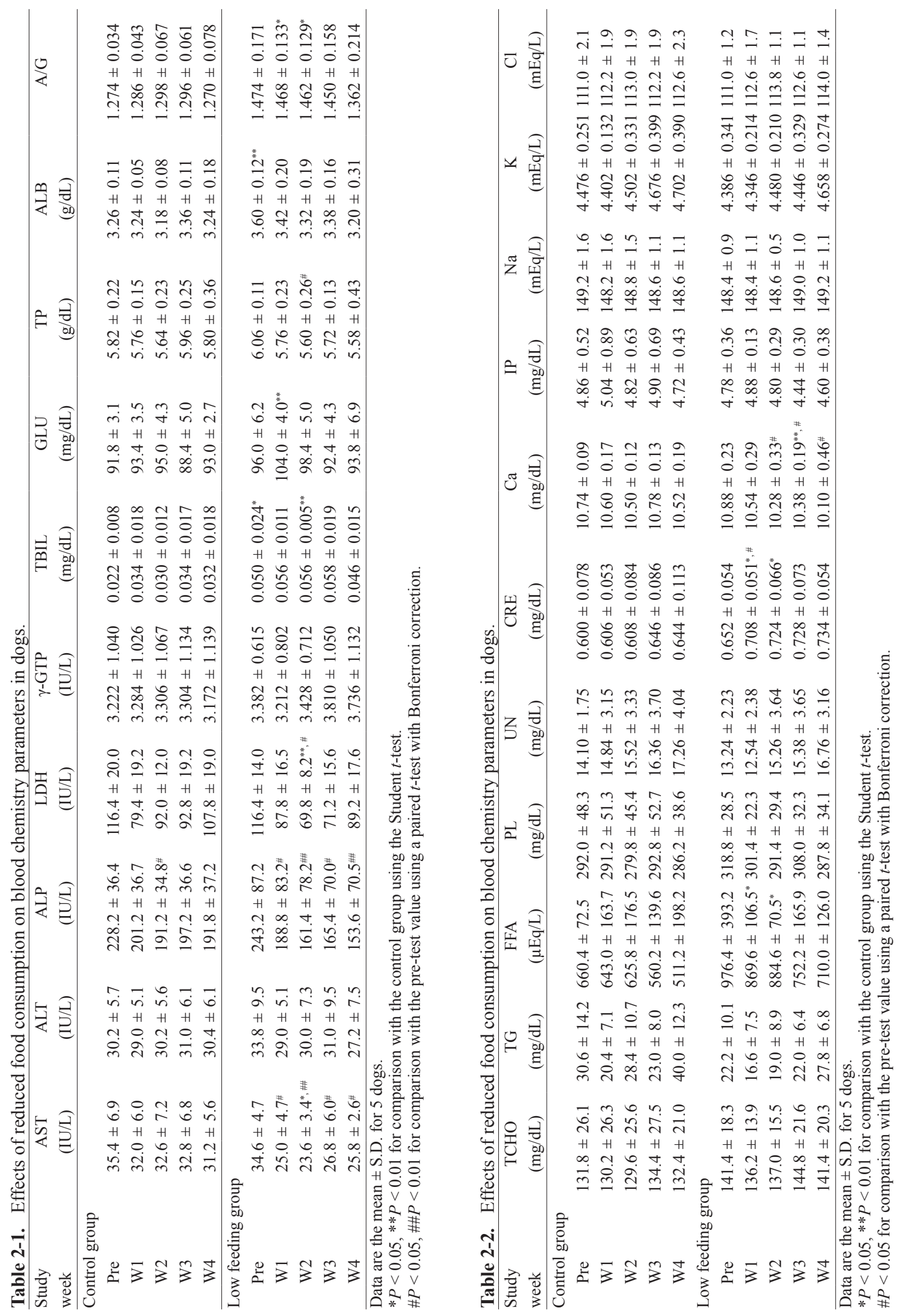

Vol. 42 No. 1 
Effects of reduced food intake in dogs

Table 3. Effects of reduced food consumption on alkaline phosphatase isozyme levels in dogs.

\begin{tabular}{lcccc}
\hline $\begin{array}{l}\text { Study } \\
\text { week }\end{array}$ & $\begin{array}{c}\text { ALP1 } \\
\text { (IU/L) }\end{array}$ & $\begin{array}{c}\text { ALP2 } \\
(\mathrm{IU} / \mathrm{L})\end{array}$ & $\begin{array}{c}\text { ALP3 } \\
(\mathrm{IU} / \mathrm{L})\end{array}$ & $\begin{array}{c}\text { ALP5 } \\
(\mathrm{IU} / \mathrm{L})\end{array}$ \\
\hline $\begin{array}{l}\text { Control group } \\
\text { Pre }\end{array}$ & & & & \\
W1 & $0.28 \pm 0.63$ & $33.44 \pm 10.23$ & $187.36 \pm 44.12$ & $8.14 \pm 6.21$ \\
W2 & $0.00 \pm 0.00$ & $28.20 \pm 14.86$ & $164.66 \pm 41.41$ & $8.56 \pm 6.65$ \\
W3 & $2.50 \pm 3.46$ & $30.74 \pm 9.31$ & $149.44 \pm 35.40$ & $5.90 \pm 5.69$ \\
W4 & $0.46 \pm 1.03$ & $32.92 \pm 12.17$ & $157.90 \pm 41.82$ & $3.60 \pm 3.08$ \\
\hline Low feeding group & $0.98 \pm 2.19$ & $33.78 \pm 9.35$ & $153.42 \pm 32.72$ & $3.02 \pm 2.79$ \\
Pre & & & & $7.54 \pm 2.90$ \\
W1 & $0.38 \pm 0.85$ & $52.64 \pm 26.20$ & $187.14 \pm 69.33$ & $4.98 \pm 3.17$ \\
W2 & $2.88 \pm 3.18$ & $38.34 \pm 19.66$ & $140.06 \pm 65.59$ \# & $1.66 \pm 0.63$ \\
W3 & $0.70 \pm 1.57$ & $35.70 \pm 15.16$ & $120.02 \pm 65.89$ \#\# \\
W4 & $0.00 \pm 0.00$ & $32.10 \pm 16.42$ & $131.60 \pm 54.64$ & $3.50 \pm 4.73$ \\
\hline
\end{tabular}

Data are the mean \pm S.D. for 5 dogs.

$\# P<0.05, \# \# P<0.01$ for comparison with the pre-test value using a paired $t$-test with Bonferroni correction.

Other values were not significantly different from the control group (Student t-test).

Table 4. Effects of reduced food consumption on electrocardiographic parameters in dogs.

\begin{tabular}{|c|c|c|c|c|c|}
\hline $\begin{array}{l}\text { Study } \\
\text { week }\end{array}$ & $\begin{array}{c}\mathrm{HR} \\
\text { (bpm) }\end{array}$ & $\begin{array}{l}\text { PR interval } \\
(\mathrm{msec})\end{array}$ & $\begin{array}{l}\text { QRS duration } \\
(\mathrm{msec})\end{array}$ & $\begin{array}{l}\text { QT interval } \\
(\mathrm{msec})\end{array}$ & QTc \\
\hline \multicolumn{6}{|c|}{ Control group } \\
\hline Pre & $102.6 \pm 9.9$ & $100.2 \pm 11.0$ & $40.0 \pm 1.7$ & $191.2 \pm 14.1$ & $192.0 \pm 14.6$ \\
\hline W1 & $104.0 \pm 12.2$ & $97.8 \pm 11.5$ & $39.8 \pm 2.5$ & $193.4 \pm 13.5$ & $194.4 \pm 10.9$ \\
\hline W2 & $100.6 \pm 8.6$ & $98.2 \pm 16.5$ & $38.4 \pm 2.8$ & $193.2 \pm 14.1$ & $193.2 \pm 12.5$ \\
\hline W3 & $95.0 \pm 13.2$ & $104.6 \pm 16.9$ & $37.8 \pm 2.2$ & $190.6 \pm 12.5$ & $188.8 \pm 9.5$ \\
\hline W4 & $98.8 \pm 9.1$ & $104.6 \pm 19.2$ & $41.4 \pm 2.6$ & $194.0 \pm 13.9$ & $193.8 \pm 13.0$ \\
\hline \multicolumn{6}{|c|}{ Low feeding group } \\
\hline Pre & $99.2 \pm 17.8$ & $100.6 \pm 13.7$ & $40.4 \pm 1.1$ & $181.4 \pm 10.9$ & $180.4 \pm 6.7$ \\
\hline W1 & $92.6 \pm 19.3$ & $103.0 \pm 13.8$ & $39.2 \pm 2.0$ & $193.8 \pm 9.4$ & $190.8 \pm 5.4$ \\
\hline W2 & $81.8 \pm 16.9$ & $105.4 \pm 10.2$ & $38.6 \pm 2.9$ & $200.2 \pm 13.3$ & $193.6 \pm 10.4$ \\
\hline W3 & $70.2 \pm 13.4^{*}, \#$ & $122.8 \pm 26.6$ & $39.8 \pm 0.4$ & $213.2 \pm 20.8$ & $201.4 \pm 16.1$ \\
\hline W4 & $66.8 \pm 21.9^{*}$ & $111.8 \pm 22.1$ & $44.2 \pm 2.2^{\#}$ & $217.0 \pm 31.4$ & $202.4 \pm 22.4$ \\
\hline
\end{tabular}

Data are the mean \pm S.D. for 5 dogs.

$* P<0.05$ for comparison with the control group using the Student $t$-test.

$\# P<0.05$ for comparison with the pre-test value using a paired $t$-test with Bonferroni correction.

\section{Electrocardiography}

Changes in the electrocardiograms are shown in Table 4. The electrocardiograms in the LF group revealed significant decreases $(P<0.05)$ in the HR at W3 and W4, compared with that in the control group and/or the pretest value. In the LF group, the average percent changes in the HR at W3 and W4 were $-29.2 \%$ and $-32.7 \%$ relative to the pre-test value, respectively. A significant prolongation $(P<0.05)$ of the QRS duration in the LF group, compared with the pre-test value, was observed at W4. In the LF group, the average percent change in the QRS duration at $\mathrm{W} 4$ was $+9.4 \%$ relative to the pre-test value. A tendency toward a prolonged PR interval was observed at W3 and W4 in the LF group, compared with that in the control group and the pre-test value. In the LF group, the average percent changes in the PR interval at W3 and W4 were $+22.1 \%$ and $+11.1 \%$ relative to the pre-test value, respectively. Furthermore, the occurrence of a second-degree atrioventricular block was observed at W3 and W4 in 2 dogs in the LF group.

\section{Circulating blood}

Changes in the circulating blood parameters are shown in Table 5. In the LF group, a significantly lower $(P<0.05)$ circulating blood volume and a tendency toward a lower circulating plasma volume, compared with that in the control group and the pre-test value, were observed at W4. In the LF group, the average percent 
J. Morita et al.

Table 5. Effects of reduced food consumption on circulating blood parameters in dogs.

\begin{tabular}{lccc}
\hline $\begin{array}{l}\text { Study } \\
\text { week }\end{array}$ & $\begin{array}{c}\text { Circulating plasma volume } \\
(\mathrm{mL})\end{array}$ & $\begin{array}{c}\text { Circulating blood volume } \\
(\mathrm{mL})\end{array}$ & $\begin{array}{c}\text { Circulating blood volume /body weight } \\
(\mathrm{mL} / \mathrm{kg})\end{array}$ \\
\hline $\begin{array}{l}\text { Control group } \\
\text { Pre }\end{array}$ & $642 \pm 63$ & $1159 \pm 100$ & $108 \pm 13$ \\
W4 & $648 \pm 57$ & $1187 \pm 116$ & $110 \pm 9$ \\
\hline Low feeding group & & & $10.76 \pm 0.71$ \\
Pre & $660 \pm 93$ & $1296 \pm 181$ & $119 \pm 18$ \\
W4 & $600 \pm 26$ & $1037 \pm 65^{*, \#}$ & $112 \pm 10$ \\
\hline
\end{tabular}

Data are the mean \pm S.D. for 5 dogs.

$* P<0.05$ for comparison with the control group using the Student $t$-test.

$\# P<0.05, \# \#<0.01$ for comparison with the pre-test value using a paired $t$-test with Bonferroni correction.
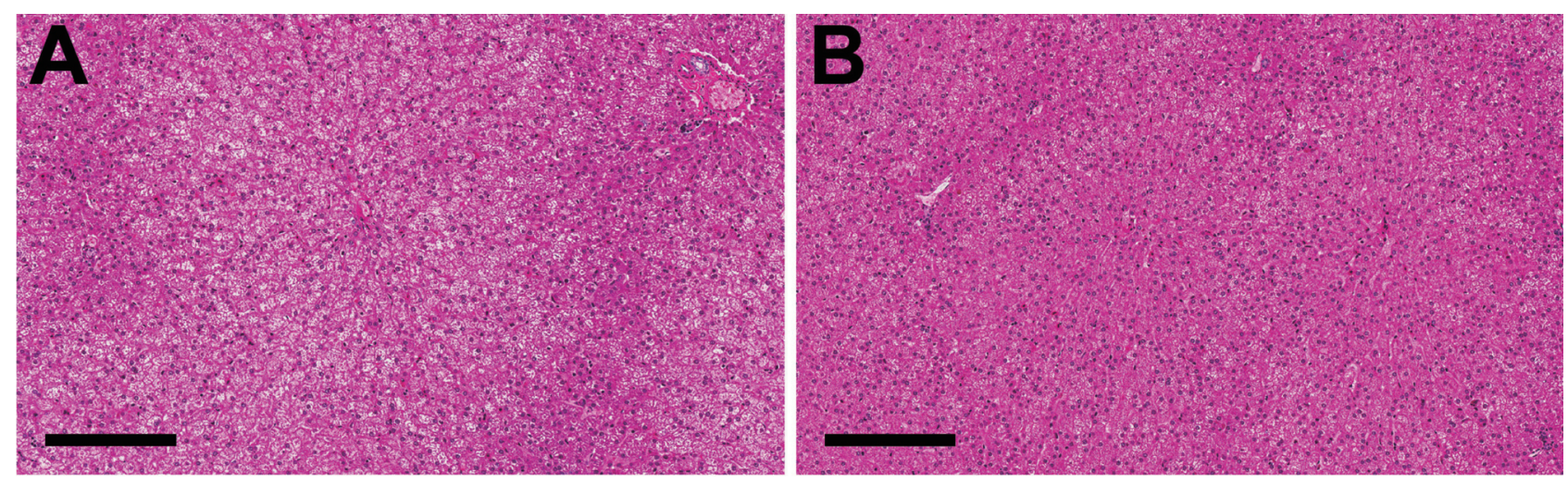

Fig. 3. Histopathological changes in the liver. Rarefaction of the cytoplasmic staining is observed in the centrilobular hepatocytes to a mild degree in the control group (A) and a minimal degree in the LF group (B). This change implies a decrease in glycogen in the hepatocytes in the LF group. H\&E staining. Bar $=200 \mu \mathrm{m}$.

changes in the circulating blood volume and the circulating plasma volume at $\mathrm{W} 4$ were $-20.0 \%$ and $-9.1 \%$ relative to the pre-test values, respectively. The circulating blood volume per body weight ratio was comparable to the pre-test value and the value observed in the control group.

\section{Organ weight and histopathology}

The organ weights are shown in Table 6. In the LF group, the weights of the liver and thymus were significantly lower $(P<0.05)$ than those in the control group. In the LF group, the average percent changes in the weights of the liver and thymus were $-21.7 \%$ and $-50.6 \%$ relative to the values in the control group, respectively.

Histopathological examinations revealed minimal hepatocyte rarefaction in all the dogs in the LF group and mild hepatocyte rarefaction in all the dogs in the control group (Fig. 3). In the thymus, mild atrophy was observed in 3 dogs in the LF group. No reduced feeding-related histopathological changes were observed in any of the other organs in any of the dogs.

\section{DISCUSSION}

In the present study, the effects of a reduced food intake for 4 weeks on physiological parameters in dogs were clarified. Decreases in the ALP3 level and the circulating blood volume as a result of changes in the physiological state of dogs subjected to reduced feeding conditions were demonstrated. The following changes were also observed in the LF group: a decrease in body weight; decreases in the WBC, Neut, RBC, and Retic counts and the HGB and HCT levels in the hematologic evaluation; decreases in the AST, ALP, LDH and Ca levels and an increase in the CRE level in the blood chemistry evaluation; a decrease in the HR, a prolonged QRS duration, and the occurrence of a second-degree atrioventricular block in the electrocardiographic evaluation; and decreases in the liver and thymus weights, a decrease in hepatocyte rarefaction, and thymic atrophy in the pathological 
Effects of reduced food intake in dogs

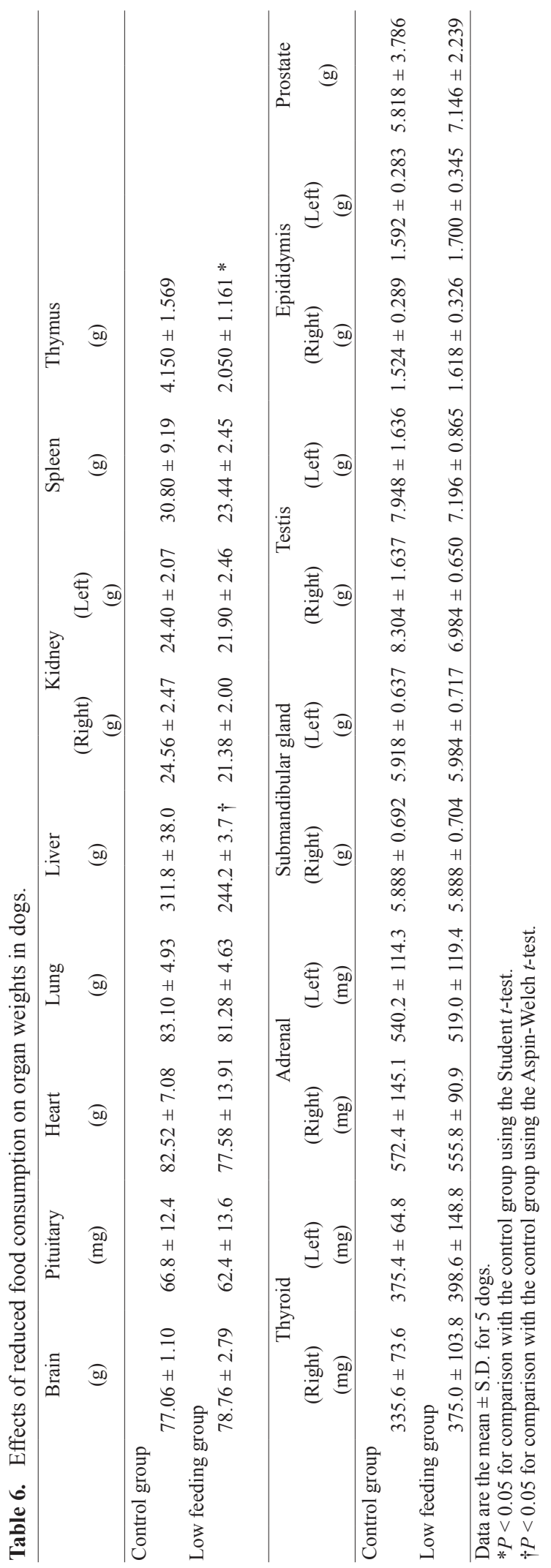


evaluation.

The blood chemistry results showed a decrease in the ALP level, similar to previously reported findings (Morita et al., 2015; Takamatsu et al., 2015). In the present study, ALP isozymes were assayed to confirm which organs were affected by the reduced food intake. A decreased ALP3 level was observed in the LF group, suggesting that the reduced food intake influenced the bone and possibly accelerated physiological changes related to bone turnover. The effects of a reduced food intake on the bone were considered to be minimal because this finding was detected as a decrease in the ALP3 level, but histopathological changes were not observed.

A decrease in the circulating blood volume was observed in the LF group. This change was thought to have arisen from the decrease in body weight caused by the reduced food intake, since the reduced feeding did not have any effect on the circulating blood volume per body weight ratio. A tendency toward a decreased circulating plasma volume was also observed in the LF group. Water intake is reportedly correlated with eating in experimental animals (Kraly, 1984), and a decrease in water consumption has been reported in dogs subjected to a reduced food intake (Takamatsu et al., 2015). The change in the circulating plasma volume was considered to have been caused by a decrease in water consumption. On the other hand, in the hematology findings, a decreased HCT level was observed under reduced feeding conditions in the LF group. Thus, the decrease in the circulating blood volume, which is composed of the circulating plasma and blood cells, was likely caused by the reduced food intake and/or body weight. These changes in circulation parameters might have influenced cardiac function, such as a decreased HR as a result of a decreased cardiac afterload.

The hematology findings showed decreases in the erythroid parameters in the LF group, and these changes were thought to be related to a decrease in erythropoiesis associated with the decrease in the Retic count caused by the reduced food intake, since no changes suggestive of erythrocyte destruction, such as hemolysis, were observed. In dogs, transient increases in the RBC count and the HGB and HCT levels, suggestive of hemoconcentration, were observed at week 4 in a previously reported reduced feeding study (Morita et al., 2015). In the present study, the hemoconcentration was also thought to have been caused by reduced food intake; however, the decrease in erythropoiesis was considered to be a stronger effect than the hemoconcentration. These variations in the erythroid parameters might be related to the energy requirements associated with physical size at the beginning of the reduced feeding period.
In the electrocardiography studies, a decrease in the HR with a subsequent prolongation of the QRS duration and a tendency toward a prolonged PR interval were observed in this study, and similar changes have been previously reported to be associated with a reduced food intake (Morita et al., 2015; Takamatsu et al., 2015) or malnutrition (Alden et al., 1987). Body weight loss associated with changes in diet reportedly affects autonomic nervous activity in dogs (Rocchini et al., 1989; Hall et al., 1993; Truett et al., 1996). In the present study, changes in autonomic nervous activity were thought to have caused the decrease in HR. Bradyarrhythmias associated with a second-degree atrioventricular block were also observed in the LF group and were considered to be related to the decreased HR and the changes in autonomic nervous activity caused by the reduced food intake.

The histopathology findings revealed minimal and mild rarefactions of the hepatocytes, implying very small and small accumulations of glycogen, in the LF and control groups, respectively. This difference in histopathological grade suggests that glycogen accumulated to a lesser degree in the hepatocytes in the LF group than in the control group, suggesting that more hepatic glycogen was consumed in the LF group to maintain glucose homeostasis. In addition, a decrease in the weight of the liver was observed in the LF group. A reduced food intake is known to affect the weight of the liver in dogs (Takamatsu et al., 2015) and rats (Schwartz et al., 1973; Oishi et al., 1979; Pickering and Pickering, 1984; Levin et al., 1993; Seki et al., 1997; Yoshii et al., 2003; Moriyama et al., 2008), and the liver weight is reportedly associated with variations in the water and glycogen contents arising from feeding in rats (Leveille and Chakrabarty, 1967; Rothacker et al., 1988). Thus, the decrease in the weight of the liver observed in the LF group in the present study was considered to be mainly related to a decrease in glycogen.

Other significant findings observed in this study were similar to those reported previously (Morita et al., 2015; Takamatsu et al., 2015). The following results have thus been reproducibly observed in reduced food intake studies: a decrease in body weight; a decrease in the WBC count (especially the Neut count); decreases in the AST, $\mathrm{LDH}$, and Ca levels; an increase in the CRE level; a decrease in the thymus weight; and mild atrophy in the thymus.

In the present study, significant decreases in body weight, erythroid parameters, and ALP3 and AST levels and a significant increase in the CRE level at W1 or thereafter and significant decreases in the Neut, LDH and $\mathrm{Ca}$ levels at W2 or thereafter were observed in the LF group. These findings were thought to be an early reflec- 
Effects of reduced food intake in dogs

tion of poor nutrient intake as a result of reduced feeding because of the high metabolic activity level. Subsequently, a significant decrease in the HR (approximately $30 \%$ below the pre-test value) was observed at W3 or thereafter in the LF group. During this same time period of W3 or thereafter, the body weight in the LF group had decreased by approximately $10 \%$. A similar tendency regarding the timing of the decrease in the HR and the loss of approximately $10 \%$ of the body weight has been observed under several reduced feeding conditions in previous reports (Morita et al., 2015; Takamatsu et al., 2015). Since body weight loss arising from a decreased food intake might contribute to the decrease in the HR, careful monitoring of the HR might be necessary if the body weight decreases by approximately $10 \%$ in general toxicity studies performed using dogs. Furthermore, bradycardia related to body weight loss might cause low frequency findings, such as the second-degree atrioventricular blocks that were observed in the LF group at W3 or thereafter.

The statistically significant changes in the MCHC in the hematologic evaluation and in the TBIL, GLU, TP, $\mathrm{A} / \mathrm{G}$ and FFA values in the blood chemistry evaluation were not considered to be correlated with the reduced food intake because these values were within the range of historical control subjects in our laboratory (data not shown) and showed very minimal changes relative to the pre-test values or the values for the control group.

An increase in the FFA level related to insufficient energy intake was observed in a previously reported reduced feeding study in dogs (Morita et al., 2015). In the present study, the FFA level during the pre-test period in the LF group was relatively higher than the control group and the previous study (Morita et al., 2015), and the production of FFA in the LF group was thought to be had a measurable influence by overnight fast. The FFA level throughout the reduced feeding period in the LF group was comparable to the pre-test value, because the level was also thought to be mainly influenced by overnight fast. The study-to-study variation in the FFA level might be related to a difference of effect of overnight fast in dogs.

In conclusion, we found that the ALP3 level and the circulating blood volume were decreased in dogs subjected to reduced feeding for 4 weeks. A decreased RBC count and HGB and HCT levels, the occurrence of second-degree atrioventricular block, and decreased hepatocyte rarefaction were also observed. These findings have not been previously reported in dogs with a decreased food intake. Furthermore, the presently observed changes in body weight, WBC (Neut) and Retic counts, AST, ALP, LDH, CRE, and Ca levels, HR, QRS duration, liver and thymus weights, and mild thymic atrophy were similar to those reported previously. These results provide useful information for assessing the safety of test compounds in toxicological studies, enabling direct treatment effects to be distinguished from secondary changes as a result of a decreased food intake.

Conflict of interest---- The authors declare that there is no conflict of interest.

\section{REFERENCES}

Alden, P.B., Madoff, R.D., Stahl, T.J., Lakatua, D.J., Ring, W.S. and Cerra, F.B. (1987): Left ventricular function in malnutrition. Am. J. Physiol., 253, H380-H387.

Durrer, J.L. and Hannon, J.P. (1962): Seasonal variations in caloric intake of dogs living in an arctic environment. Am. J. Physiol., 202, 375-378.

Feldschuh, J. and Enson, Y. (1977): Prediction of the normal blood volume. Relation of blood volume to body habitus. Circulation, 56, 605-612.

Hall, J.E., Brands, M.W., Dixon, W.N. and Smith, M.J.Jr. (1993): Obesity-induced hypertension. Renal function and systemic hemodynamics. Hypertension, 22, 292-299.

Kraly, F.S. (1984): Physiology of drinking elicited by eating. Psychol. Rev., 91, 478-490.

Leveille, G.A. and Chakrabarty, K. (1967): Diurnal variations in tissue glycogen and liver weight of meal-fed rats. J. Nutr., 93, 546554.

Levin, S., Semler, D. and Ruben, Z. (1993): Effects of two weeks of feed restriction on some common toxicologic parameters in Sprague-Dawley rats. Toxicol. Pathol., 21, 1-14.

Matsunaga, T., Mitsui, T., Harada, T., Inokuma, M., Murano, H. and Shibutani, Y. (1997): QT corrected for heart rate and relation between QT and RR intervals in beagle dogs. J. Pharmacol. Toxicol. Methods, 38, 201-209.

Morita, J., Izumi, T., Sunouchi, M., Arima, K. and Tsutsumi. S., (2015): Effects of reduced food intake on the parameters of toxicity evaluation in dogs. J. Toxicol. Sci., 40, 523-533.

Moriyama, T., Tsujioka, S., Ohira, T., Nonaka, S., Ikeda, H., Sugiura, H., Tomohiro, M., Samura, K. and Nishikibe, M. (2008): Effects of reduced food intake on toxicity study parameters in rats. J. Toxicol. Sci., 33, 537-547.

Oishi, S., Oishi, H. and Hiraga, K. (1979): The effect of food restriction for 4 weeks on common toxicity parameters in male rats. Toxicol. Appl. Pharmacol., 47, 15-22.

Pickering, R.G. and Pickering, C.E. (1984): The effects of reduced dietary intake upon the body and organ weights, and some clinical chemistry and haematological variates of the young Wistar rat. Toxicol. Lett., 21, 271-277.

Rocchini, A.P., Key, J., Bondie, D., Chico, R., Moorehead, C., Katch, V. and Martin, M. (1989): The effect of weight loss on the sensitivity of blood pressure to sodium in obese adolescents. N. Engl. J. Med., 321, 580-585.

Rothacker, D.L., Kanerva, R.L., Wyder, W.E., Alden, C.L. and Maurer, J.K. (1988): Effects of variation of necropsy time and fasting on liver weights and liver components in rats. Toxicol. Pathol., 16, 22-26.

Schwartz, E., Tornaben, J.A. and Boxill, G.C. (1973): The effects of 


\section{J. Morita et al.}

food restriction on hematology, clinical chemistry and pathology in the albino rat. Toxicol. Appl. Pharmacol., 25, 515-524.

Seki, M., Yamaguchi, K., Marumo, H. and Imai, K. (1997): Effects of food restriction on reproductive and toxicological parameters in rats - in search of suitable feeding regimen in long-term tests. J. Toxicol. Sci., 22, 427-437.

Takamatsu, K., Yamashita, H., Satake, S., Kazusa, K., Tabata, H. and Nishikata, T. (2015): Effects of four-week feed restriction on toxicological parameters in beagle dogs. Exp. Anim., 64, 269280.

Truett, A.A., Borne, A.T., Poincot, M.A. and West, D.B. (1996):
Autonomic control of blood pressure and heart rate in obese hypertensive dogs. Am. J. Physiol., 270, R541-R549.

Yoshii, A., Shiraishi, Y., Ogawa, S., Kinomoto, T., Iino, Y., Matsui, A., Sawada, M., Hamano, H., Kuroda, H., Hayashi, Y., Nishi, N., Mera, Y. and Takei, M. (2003): Effects of food restriction on result of hematology examination and urinalysis in $\mathrm{CD}(\mathrm{SD})$ IGS rat -a four-week restricted feeding examination with two-week recovery period in six-week-old rats-. In Biological Reference Data on CD(SD)IGS Rats- 2002/2003 (Maeda, Y. and Shibuya, K., ed.), pp.73-84. Best Printing, Tokyo. 